\title{
河口混合过程的研究 *
}

\section{周济福 刘青泉 李家春}

(中国科学院力学研究所, 北京 10008 ;)

\begin{abstract}
摘要 根据河口水流运动特点, 应用平板振荡边界层理论及波流分解方法, 导出了 往复运动水流的流速垂向结构, 据此建立了河口准二维盐度数学模型, 并得到实测资 料验证. 应用该堆型研究河口混合过程, 得到了盐度分布、盐度锋强度随径流和潮差

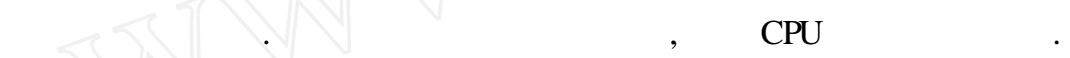

关键词 河口混合 振荡边界层 径流潮流分解 盐度

河口为盐淡水交会处, 河口混合通过絮凝影响泥沙输运 ${ }^{[1]}$, 是形成拦门沙的原因之一。盐 水入侵也是河口地区农田水利、土壤改良、城市供水等问题中不可忽视的因素. 因此,河口混 合的研究越来越受到人们的关注.

河口混合过程因径流和潮流的相对强弱而异, 大体上可分为 3 种类型, 即弱混合型、缓混 合型和强混合型。在弱混合型河口, 潮差较小, 径流作用强, 表底层盐度差别很大, 往往出现盐 水楔而形成分层流动, 现有研究主要是建立盐水楔的几何尺寸和位置与径流、潮流强度之间的 关系, 以及探讨盐淡水交界面掺混过程的机理 (Grubert ${ }^{[2,3]}$ 和 Kurup ${ }^{[4]}$ ); 而在强混合型河口, 潮 差较大, 潮流作用强, 盐度沿垂线几乎均匀分布, 可用较简单的模式或一维数学模型进行求解; 韩曾萃等人 ${ }^{[5]}$ 曾用一维水量、动量和盐量平衡方程模拟钱塘江河口的盐水入侵;缓混合型河 口, 盐度的分布特征则介于两者之间, 三维特性较明显, 流动的垂向结构和盐度的垂向分布变 得重要,应通过动力学方法求解. 长江口即属于这一类型的河口.

严格地说, 河口水流及标量 (如泥沙、盐度等) 输运都应采用完整的三维数学模型, 如:匡翠 萍 $^{1)}$ 曾运用三维数学模型对长江口水流泥沙盐度进行模拟. 但因其涉及物理因素多, 不易分 析机理,为避免计算量过大, 垂直方向网格划分不细亦会带来误差. 因此, 目前实际应用中仍 普遍采用二维数学模型 ${ }^{[6]}$ 或准三维模型. 如 Jin 等人 ${ }^{[7]}$ 建立准三维耦合模式模拟浅水环流; Irene 等人 ${ }^{[8]}$ 在对数速度剖面的假定下,用渐近方法模拟波流共同作用下的三维泥沙输运. 这 些研究主要从计算方法上进行简化, 减少了计算工作量, 而对物理机理的考虑不够. 如, 对河 口往复运动的水流,对数速度剖面近似在转流时刻附近显然是不合理的. Collins 等人 ${ }^{[9]}$ 分析 Loughor 河口及 Swansea 海湾的潮间带实测流速剖面认为, 符合对数流速分布的数据所占比例 不足 $40 \%$,由此可见, 对河口水流完全采用对数律来近似过于粗粘.

1999-02-24 收稿, 1999-05-18 收修改稿

*国家自然科学基金(批准号:59879025)、空气动力学流体力学国家科技部攀登项目和中国科学院力学研究所所长择优 基金资助项目

1) 匡翠萍. 长江口拦门沙冲淤及悬沙沉降规律研究和水流盐度泥沙数学模型. 南京水利科学研究院博士学位论文. 1993 
本文着重研究河口水流的物理特性, 利用振荡边界层理论及径流潮流分解方法, 获得水流 垂向结构, 从而可较好地模拟盐度的对流扩散过程. 然后, 用长江口北槽的实测数据验证了所 建立的模型. 最后, 以此模型讨论河口盐淡水混合的基本规律.

\section{1 河口水流的垂向结构}

\section{1 河口水流特征}

在河口地区, 潮汐使得水流做往复运动, 因而流涑随邖间周期变化, 其周期与潮汐相同. 在落急时刻, 流速达最大值, 此时水面及垂问平均流速分别用 $u_{\max }$ 和 $U_{\max }$ 表示; 涨急时刻, 流速 达最小值 (负向最大)，此时水面及垂向平均流䢡分别用 $u_{\mathrm{min}}$ 和 $U_{\min }$ 表示. 由于潮汐的周期很 长(通常 12 或 $24 \mathrm{~h}$ ), 在涨落急时刻前后水流的运动特征与明渠单向水流极为相似. 在涨落息 时刻附近，则国水流转向，往往呈现出上下水流反向运动的特点. 径流的作用使河口水流在往 复运动的同矿, 佇在着向海的净通量, 因此一般情况下 $u_{\text {max }}^{\zeta}>-u_{\min }^{\zeta}, U_{\max }>-U_{\min }$. 为便于分 析 , 将径流和潮流的作用进行分解, 即

$$
\begin{gathered}
U=U_{\mathrm{c}}+U_{\mathrm{w}}, \\
u(z)=u_{\mathrm{c}}(z)+u_{\mathrm{w}}(z) .
\end{gathered}
$$

这里 $U$ 为垂向平均流速, $u(z)$ 为垂直坐标 $z$ 处的水平流速, $U_{\mathrm{w}}$ 和 $U_{\mathrm{c}}$ 分别表示 $U$ 的潮流径流 分量, $u_{\mathrm{w}}(z)$ 和 $u_{\mathrm{c}}(z)$ 分别表示 $u(z)$ 的潮流径流分量. $u_{\mathrm{w}}(z)$ 和 $u_{\mathrm{c}}(z)$ 可分别看做水面流速的 波流分量 $u_{\mathrm{w}}^{\zeta}$ 和 $u_{\mathrm{c}}$ 的函数, 因为 $u_{\mathrm{w}}(z)$ 可由振荡边界层理论与 $u_{\mathrm{w}}$ 建立关系, 而单向水流的对 数剖面近似在工程实际中具有足够的精度, 它可将 $u_{\mathrm{c}}(z)$ 与 $u_{\mathrm{c}}$ 联系起来. 为此定义

$$
\begin{aligned}
& u_{\mathrm{c}}=\frac{1}{2}\left[u_{\max }+\zeta_{\min }\right], \\
& u_{\mathrm{w}}=\frac{1}{2}\left[\zeta_{\max }-u_{\min }\right] \cos \phi,
\end{aligned}
$$

其中 $\phi$ 为相位. 如前所述, 涨落急时刻的水流运动特征接近明渠单向流, 可用对数律近似表 示其速度剖面, 此时水面速度与平均流速的关系为

$$
u_{\max }=U_{\max }\left(\begin{array}{l}
1+\frac{n \sqrt{g}}{\mathrm{~K} h_{\max }^{1 / 6}} \\
u_{\min }=U_{\min } \\
1+\frac{n \sqrt{g}}{\mathrm{~K} h_{\min }^{1 / 6}}
\end{array}\right),
$$

其中 $\mathrm{K}$ 为 Karman 常数, $n$ 为 Manning 糙率系数, $g$ 为重力加速度, $h_{\max }$ 和 $h_{\min }$ 分别为相应于 $U_{\max }$ 和 $U_{\mathrm{min}}$ 的水深, 即落急、涨急时刻的水深.

\section{2 流速垂向结构}

虽然浅水运动水平流速几乎均匀,用一维方程即可得到平均流场, 但在研究盐度分布时必 须了解其垂向结构才能正确描述其对流扩散过程. 如上所述水流运动特征与振荡边界层相 似, 因此可以利用平板振荡边界层理论, 辅之以径流潮流分解, 来模拟河口水流的垂向二维结 构,这样,可在仅知某一坐标 $x$ 处的垂线平均流速的时间过程 $U$ 的条件下,得到水平流速的垂 线分布 $u(z)$,并导出垂向速度 $w(z)$ 的分布. 
首先由对数律推导径流分量 $u_{\mathrm{c}}(z)$ 与 $u_{\mathrm{c}}$ 的关系. 根据对数流速分布, 成立下式

$$
\frac{u_{\mathrm{c}}-u_{\mathrm{c}}(z)}{u *_{\mathrm{c}}}=\frac{1}{\mathrm{~K}} \ln \frac{h}{z},
$$

其中 $u *_{c}$ 为对应于径流分量的摩擦速度, 作垂向平均后可得

$$
\begin{gathered}
u_{\mathrm{c}}=U_{\mathrm{c}}+\frac{u *_{\mathrm{c}}}{\mathrm{K}}, \\
u *_{\mathrm{c}}=n \sqrt{g} U_{\mathrm{c}} / h^{1 / 6} .
\end{gathered}
$$

将(3) (5) 式联解得

$$
u_{\mathrm{c}}(z)=u_{\mathrm{c}}\left(1-\frac{1}{1+\frac{\mathrm{K} h^{1 / 6}}{n \sqrt{g}}} \ln \frac{h}{z}\right) .
$$

其次根据振荡边界层理论推导潮流分量 $u_{\mathrm{w}}(z)$ 与 $u_{\mathrm{w}}$ 的关系. 当坐标系固定于底床时, 水 面速度以 $u_{\mathrm{w}}^{\zeta}=\frac{1}{2}\left[\zeta_{\max }^{\zeta}-\zeta_{\min }^{\zeta}\right] \cos \phi=A \cos \phi$ 做简谐振荡, 将因粘性应力的作用而导致 $z$ 方向上 的每层流体在 $x$ 方向做相应的振荡运动, 其速度分布为 ${ }^{[10]}$

$$
u_{\mathrm{w}}(z)=A \cos \phi-A \exp \left(-\left(\frac{z}{\delta}\right)^{p}\right) \cos \left(\left(\frac{z}{\delta}\right)^{p}-\phi\right) \text {, }
$$

其中 $\delta=\sqrt{\frac{2}{\omega}}$ 称为穿透深度, $\mathrm{v}$ 为流体的粘性系数, $\omega$ 是平板的振荡频率, $A$ 为振幅, $p$ 为一常 数, 在湍流情况下可取为 $\frac{1}{3}$, 相位 $\phi$ 是未知的, 它应满足 $U_{\mathrm{w}}=\frac{1}{h} \int_{0}^{h} u_{\mathrm{w}}(z) \mathrm{d} z$, 式中 $U_{\mathrm{w}}=U-$ $U_{\mathrm{c}}, U_{\mathrm{c}}$ 可由 (4),(5) 式解出. 将(7)式代入, 积分得

$$
\begin{aligned}
& U_{\mathrm{w}}=\sqrt{(A+\mathrm{\alpha})^{2}+\beta^{2} \sin (\phi+\psi),} \\
& \phi=\arcsin \frac{U_{\mathrm{w}}}{\sqrt{(A+\mathrm{a})^{2}+\beta^{2}}}-\psi,
\end{aligned}
$$

其中 $\psi=\arctan \frac{A+\alpha}{\beta}$ ，

$$
\begin{aligned}
& \alpha=\frac{\Delta \delta}{2 p h}\left\{\exp \left(-\left(\frac{h}{\delta}\right)^{p}\right)\left[\left(\frac{h}{\delta}\right)^{p}+1\right]\left[\left(\left(\frac{h}{\delta}\right)^{p}-1\right) \cos \left(\frac{h}{\delta}\right)^{p}-\left(\left\{\frac{h}{\delta}\right)^{p}+1\right) \sin \left(\frac{h}{\delta}\right)^{p}+1\right\},\right. \\
& \beta=\frac{\Delta \delta}{2 p h}\left\{\exp \left(-\left(\frac{h}{\delta}\right)^{p}\right)\left[\left(\frac{h}{\delta}\right)^{p}+1\right]\left[\left(\left[\frac{h}{\delta}\right)^{p}+1\right) \cos \left(\frac{h}{\delta}\right)^{p}+\left(\left\{\frac{h}{\delta}\right)^{p}-1\right) \sin \left(\frac{h}{\delta}\right)^{p}\right]-1\right\} .
\end{aligned}
$$

上述推导通过 $(6) \sim(8)$ 式建立了 $u(z)$ 与 $U$ 的关系, 这样通过平均速度即可得到流速的径流 潮流分量的垂向分布,进而可得二维流场.

\section{2 垂向二维模型及其验证}

2.1 基本控制方程

一维水流连续方程

$$
\frac{\partial s}{\partial t}+\frac{\partial U h}{\partial x}=0
$$


一维水流运动方程

$$
\frac{\partial U}{\partial t}+U \frac{\partial U}{\partial x}=-g \frac{\partial \zeta}{\partial x}-\frac{g h}{\rho} \frac{\partial \bar{p}}{\partial x}-g \frac{|U| U}{C^{2} h},
$$

状态方程

$$
\rho=\rho_{0}+\eta s,
$$

垂向二维水流连续方程

$$
\frac{\partial u}{\partial x}+\frac{\partial w}{\partial z}=0
$$

垂向二维盐度对流扩散方程

$$
\frac{\partial s}{\partial t}+\frac{\partial u s}{\partial x}+\frac{\partial w s}{\partial z}=\frac{\partial}{\partial x}\left(D_{x} \frac{\partial s}{\partial x}\right)+\frac{\partial}{\partial z}\left(D_{z} \frac{\partial s}{\partial z}\right) .
$$

以上诸式中, $t$ 为时间变量, $x, z$ 分别为水平 (向海) 和垂直 (向上) 方向的坐标, $\zeta$ 为水位, $h$ 为水深, $C$ 为谢才系数 $\rho, \rho_{0}$ 分别为盐水和淡水的密度 $\bar{\rho}$ 为沿水深积分的平均密度,$s$ 为盐 度, $\eta$ 为常数, 当 $s$ 的单位为\%时, $\eta$ 约为 $0.0007, u, w$ 分别为 $x, z$ 方向的速度分量, $D_{x}, D_{z}$ 分 别为水平、垂直方向的紊动扩散系数.

\section{2 求解步骤及方法}

求解步骤:首先联解运动方程 $(9) \sim(11)$, 得到平均流速 $U$ 和水位了在一个潮周期内的变 化过程;利用 (6) (8) 式可得到 $u(z)$; 由连续性方程 (12) 得出 $w(z)$; 最后求解盐度的对流扩 散方程(13)，可获得二维盐度分布. 由于方程(11)中含未知量 $s$, 计算需迭代进行.

求解方法:用 Preissmann 四点偏心差分格式对方程 (9), (10) 进行离散求解。这是目前国 内外广泛采用的方法,具有很好的稳定性,计算速度也较快。解盐度对流扩散方程时,采用 ADI 法和迎风格式进行离散,所得差分方程用追赶法求解.

\section{3 模型检验}

根据长江口北槽 1989 年 9 月 3 5 日的实测潮位、盐度对模型进行了验证。潮位沿江而 下有横沙、北槽中、九段东 3 站 3 5 日逐时的实测值，横沙与九段东两站之间相距约 $40 \mathrm{~km}$, 北 槽中站距横沙约 $18 \mathrm{~km}$;盐度的测点为 $\mathrm{SN} 2, \mathrm{SN} 3, \mathrm{SN} 4$, 它们分别在横沙站下游约 $8,12,16 \mathrm{~km}$. 首先以横沙为上边界,九段东为下边界, 北槽中为验证点, 对水流模型进行验证. 计算网格为 水平方向 $\Delta x=2 \mathrm{~km}, 21$ 个网格点, 垂向分为 10 层, 11 个网格点(包括底床和水面) $\Delta t=60 \mathrm{~s}$, 䊁率系数 $n=0.01$. 上下边界均给定 9 月 4 日 6 时 $~ 5$ 日 6 时的 $24 \mathrm{~h}$ 潮位过程,初始条件给定

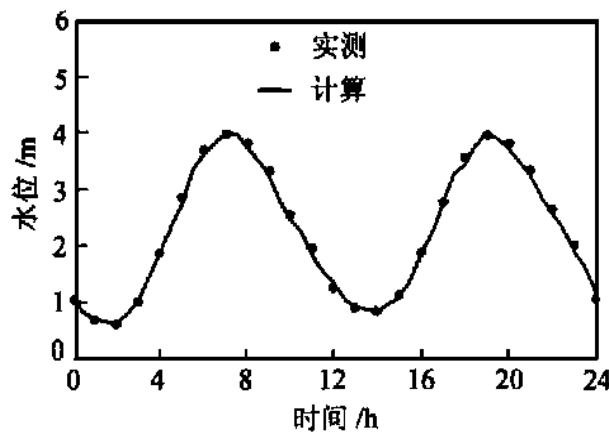

图 1 北槽中站潮位验证

各断面的水位 $\zeta(x, 0)=0$ 和各网格点的流速 $U(x, z$, $0)=0$. 计算表明, 只需 $3 \mathrm{~d}$ 便可达到稳定. 图 1 为北 槽中站计算与实测潮位的对比,两者吻合良好。图 2 为涨急、涨的时刻附近垂直剖面的流场,图示速度矢 量表明水平分量比垂直分量要大得多,这可从量级分 析得到映证. 若水平和垂直分速的特征量为 $U, W$, 水平和垂直方向的长度尺度为 $L, H$,由连续方程 (12) 应有 $W \approx U H / L$, 在本问题中, $H \approx 10 \mathrm{~m}, L \approx 40000 \mathrm{~m}$, 因此 $W \approx 2.5 \times 10^{-4} \mathrm{U}$, 说明图示流场基本反映了真实 
流动图案.

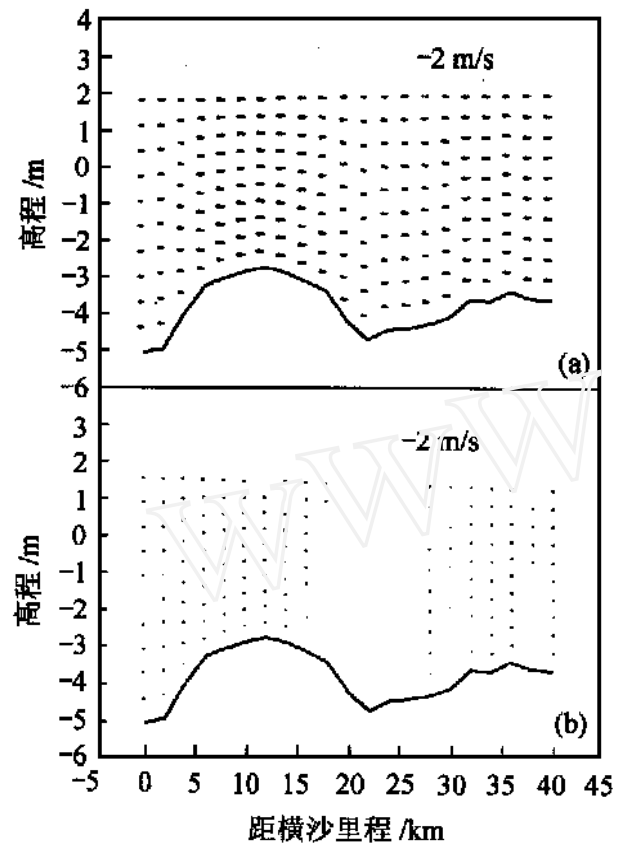

图 2 北槽涨急 (a) 涨毠 (b) 时刻的流场

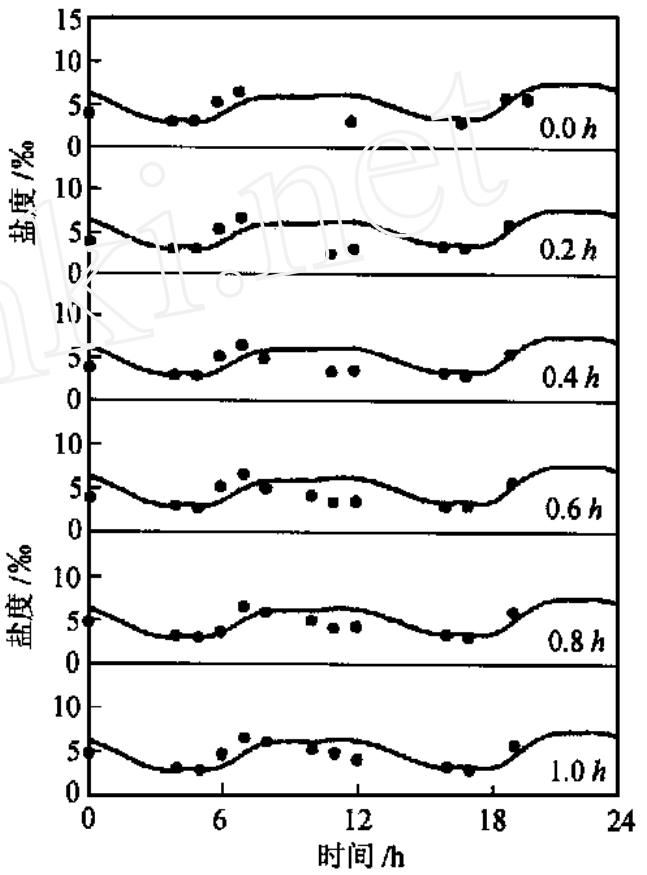

图 $3 \mathrm{SN} 3$ 测点计算和实测盐度的对比图

由于九段东站无盐度资料,盐度验证的下边界上移到 SN4 测点, 该测点处的潮位则由水 流验证时得到. 上边界仍取在横沙,文献 [11]分析了南槽的水化学成分 (包括盐度) 及其变化 规律,表明中沙灯浮(距河口的距离与横沙相当)处,年平均盐度小于 $2 \%$,该盐度值通常作为 盐淡水分界的标准. 因此, 盐度的上边界可取为零. 参照脚注 835 页 ${ }^{1)}$, 取水平扩散系数 $D_{x}=$ $500 \mathrm{~m}^{2} / \mathrm{s}$,垂向扩散系数

$$
D_{z}= \begin{cases}\mathrm{K} u * z\left(1-\frac{z}{h}\right), & \frac{z}{h}<0.5, \\ \frac{1}{4} \mathrm{~K} u * h, & \frac{z}{h} \geq 0.5,\end{cases}
$$

$u$ *为摩擦速度,其他计算参数与水流验证时相同. 初始条件 $s(x, z, 0)=0$,计算几个潮周期 后便可得到稳定的结果. 图 3 为 SN3 测点的计算 (线) 与实测 (点) 分层盐度的对比, 图示计算 结果与实测数据趋向基本吻合, 误差在工程允许的范围以内.

\section{3 径潮流对盐淡水混合的影响}

河口地区,各种动力因素以及地形的相互耦合作用,常常使混合过程变得非常复杂. 这里 仅考虑地形为平坦的情况, 以便于分析径流与潮流对混合过程的影响. 所选的参数以长江口 北橝航道为背景, 如: 水深为理论基面以下 $10 \mathrm{~m}$,并取以下几种水文条件的组合 (见表 1). 
表 1 算例表

\begin{tabular}{|c|c|c|c|c|c|}
\hline \multirow{2}{*}{ 算例 } & \multicolumn{2}{|c|}{ 计算条件 } & \multirow{2}{*}{$N$} & \multicolumn{2}{|c|}{$\frac{\Delta_{s}}{\Delta \Delta_{x}} / \mathrm{km}^{-1}$} \\
\hline & $t e / \mathrm{m}$ & $u c / \mathrm{m} \mathrm{s}^{-1}$ & & 涨憩 & 落䕀 \\
\hline (a) & 2.0 & 0.1 & 0.13 & 0.48 & 0.73 \\
\hline (b) & 1.0 & 0.1 & 0.18 & 0.61 & 0.86 \\
\hline (c) & 0.5 & 0.1 & 0.22 & 0.69 & 1.03 \\
\hline (d) & 0.5 & 0.2 & 0.40 & 1.09 & 2. 11 \\
\hline (e) & 0.5 & 0.3 & 0.57 & 1.50 & 3.10 \\
\hline
\end{tabular}

表中 $t e$ 表示潮位振幅, 即潮差之半; $u c$ 表示上边界的径流速度. 计算的河口掺混系数 $N$ 亦列于算例表 ( $N$ 为涨潮期内径流总量与涨潮总量之比,表示径流与潮流的相对强弱).

\section{1 平均盐度的水平分布}

根据以上算例可分析盐度随潮差和径流变化的规律 (图 4), 如 : (a), (b), (c) 3 种情况比 较可反映盐度随潮差变化的规律, (c), (d), (e) 3 种情况对比可分析盐度随径流变化的规律. 从图 4 可见, 盐度水平分布随时间变化, 涨憩时海水上溯最远, 盐度梯度最小;落息时径流将海

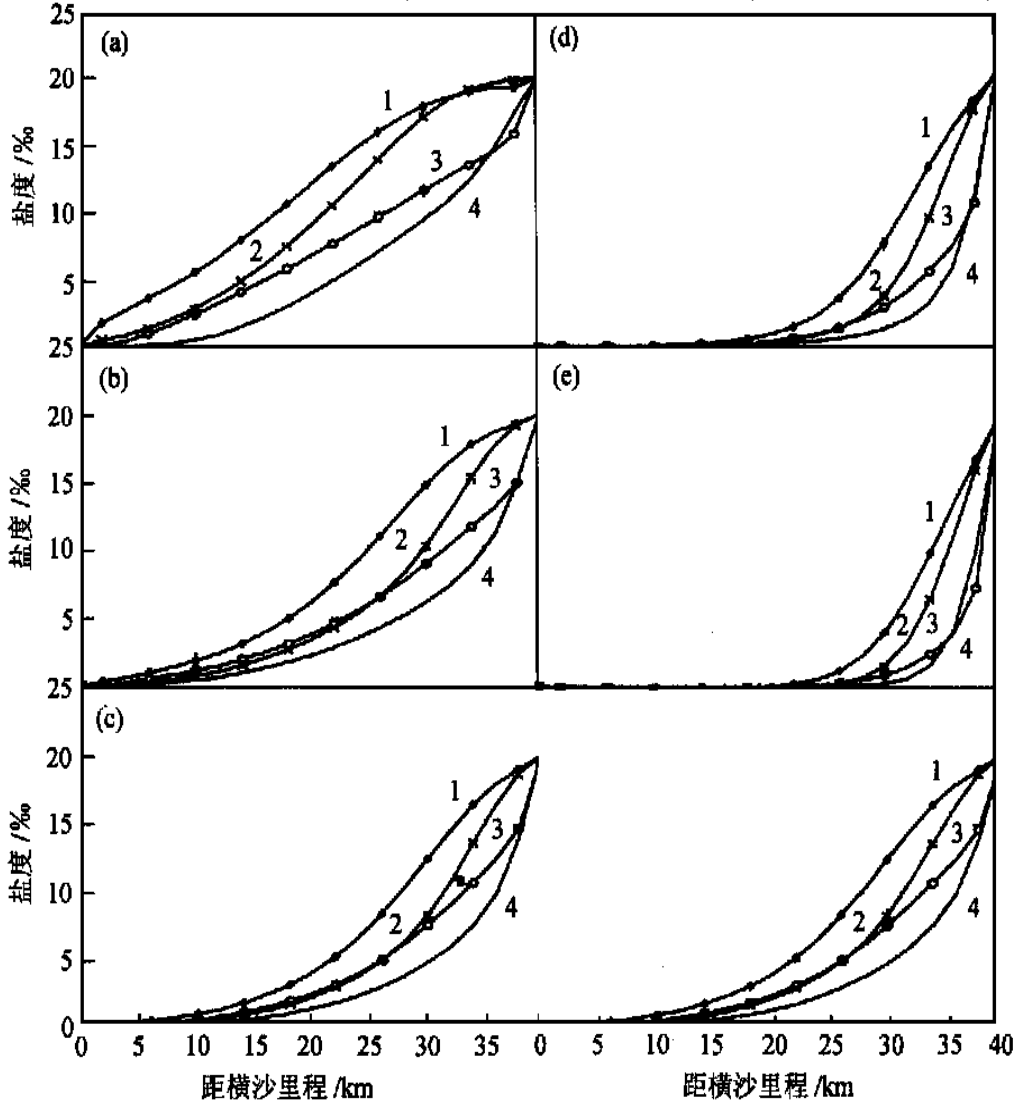

图 4 平均盐度水平分布随径流和潮差的变化

(a) $t e=2.0, u c=0.1$; (b) $t e=1.0, u c=0.1$; (c) $t e=0.5, u c=0.1$; (d) te $=0.5, u c=0.2$;

(e) $t e=0.5, u c=0.3 .1$ 一涨憩附近; 2 一涨急附近; 3 一落急附近 ; 4 一落憩附近 
水推至最下游,盐度梯度最大; 其他时刻介于两者之间。同一时刻, 当径流不变时, 随着潮差增 大, 潮流的动力作用加强, 盐度水平分布渐趋平缓. 在 $t e=2.0 \mathrm{~m}, u c=0.1 \mathrm{~m} / \mathrm{s}$ 的情况下, 盐度 在纵向剖面内接近于线性分布. 同一时刻, 当潮差不变时, 径流的加强使盐度梯度增大, 趋于 使盐度在纵向偏离线性分布. 实际上,同时刻盐度的水平分布与径流和潮差的关系可以统一 到与掺混系数的相关, (a) (e) 5 种情况由强混合转变到弱混合, 掺混系数依次增大, 与此相 应, 图示同时刻的盐度水平梯度依次增大. 当掺混系数为 0.13 时, 盐度水平分布接近于直线.

\section{2 盐度锋的变化规律}

“锋”的概念最早用在气象学上,表示两个不同温度出气团之交界面. 海洋学上也已广泛 采用这一概念,表示毗邻的两个不同性质的水河之交界面,在该交界面附近,各水文要素(盐 度、密度等)的梯度治大，河口盐度钫附近盐度的水平梯度和垂向梯度均很大，在此区域，一方 面盐度有和涩沙的䋈凝沉降, 另一方面因密度差形成的重力环流有利于泥沙的聚集, 此两方 面的效果可促使河口区 最大浑浊带”的形成.

盐度锋强度可用盐度在水平方向的梯度 $\Delta_{s} / \Delta x$ 表示,算例表中列出了各种情况下涨皕和 落毠时刻的平均盐度锋强度. 易见 $\Delta s / \Delta x$ 值随 $N$ 的 增大而增大, 即径流越大, 潮流越小时, 盐度锋强度 越大, 此时盐淡水混合较弱. 由此可以推知, 盐度锋 强度洪季大于枯季. 由于落潮时径流使盐度锋下 移, 涨潮时潮流使盐度锋上溯, 盐度锋强度落潮大于 涨潮,这一点也可从图 4 看出. 点绘 $\Delta s / \Delta x$ 和 $N$ 的 关系如图 5 ,发现涨落息时刻盐度锋强度随混合指标 基本上成线性变化,不同的时刻直线的斜率不同,从 涨㕰时刻到落㕰时刻,斜率从最小值逐渐变化到最 大值.

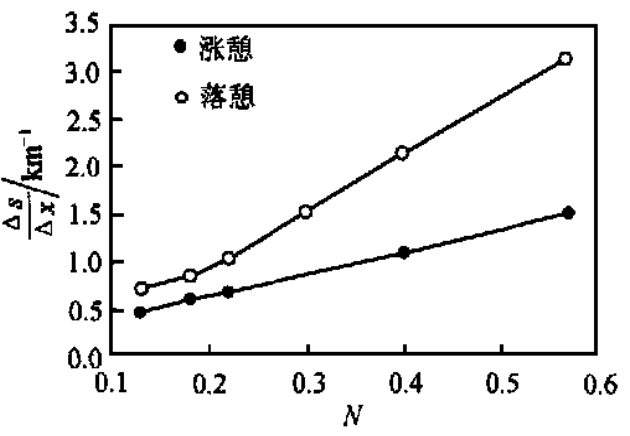

图 5 盐度锋强度与掺混系数的关系

现场观测表明, 在河口区常年存在一个水平盐度梯度最大的锋面, 其强度和位置随时间和 水文条件变化. 这一点从图 4 所示的计算结果不难看出, 如: 涨惒时刻从 (a) 到 (e), 该锋面处 的最大盐度梯度分别为 $0.70,0.94,1.00,1.37,1.78 \mathrm{~km}^{-1}$, 其位置分别在横沙下游 $20,25,28$, $32,36 \mathrm{~km}$ 附近, 随水文条件变化而迁移的幅度约 $16 \mathrm{~km}$.

\section{3 垂直剖面内盐度分布特征}

在铅垂剖面内的盐度分布等值线反映了盐度沿流向和垂向分布的概貌, 它在不同的水文 条件下表现出不同的特征,在相同的水文条件下也随时间周期地变化. 图 6(a) (d) 绘出了 $t e=1.0 \mathrm{~m}, u c=0.1 \mathrm{~m} / \mathrm{s}$ 的条件下, 涨落急、涨落媳附近的盐度分布, 从另一角度反映出毠急 时刻盐度水平分布的特点, 即盐度锋面在涨㮩时刻因潮流作用上溯到上游最远端, 而在落㮩时 刻又被径流推至最下游。同时从垂向上,可以看到在下游端出现盐水楔的维形,这是异重流运 动的必然结果. 在上游海水未侵区和下游淡水不及处, 盐度垂向分布均匀. 因此, 盐度的垂向 梯度从下游往上游是由小到大、再由大到小变化的, 即盐度垂向分布两端均匀, 中间有垂向梯 度.

图 6(f)，(d)，(e) 和 (f)，(g)，(h) 对比分别反映了径流不变而潮差分别为 $0.5,1.0,2.0 \mathrm{~m}$ 以及潮差不变径流分别为 $0.1 \mathrm{~m} / \mathrm{s}, 0.2 \mathrm{~m} / \mathrm{s}, 0.3 \mathrm{~m} / \mathrm{s}$ 时, 盐度分布的情况. 可见, 当径流不变 


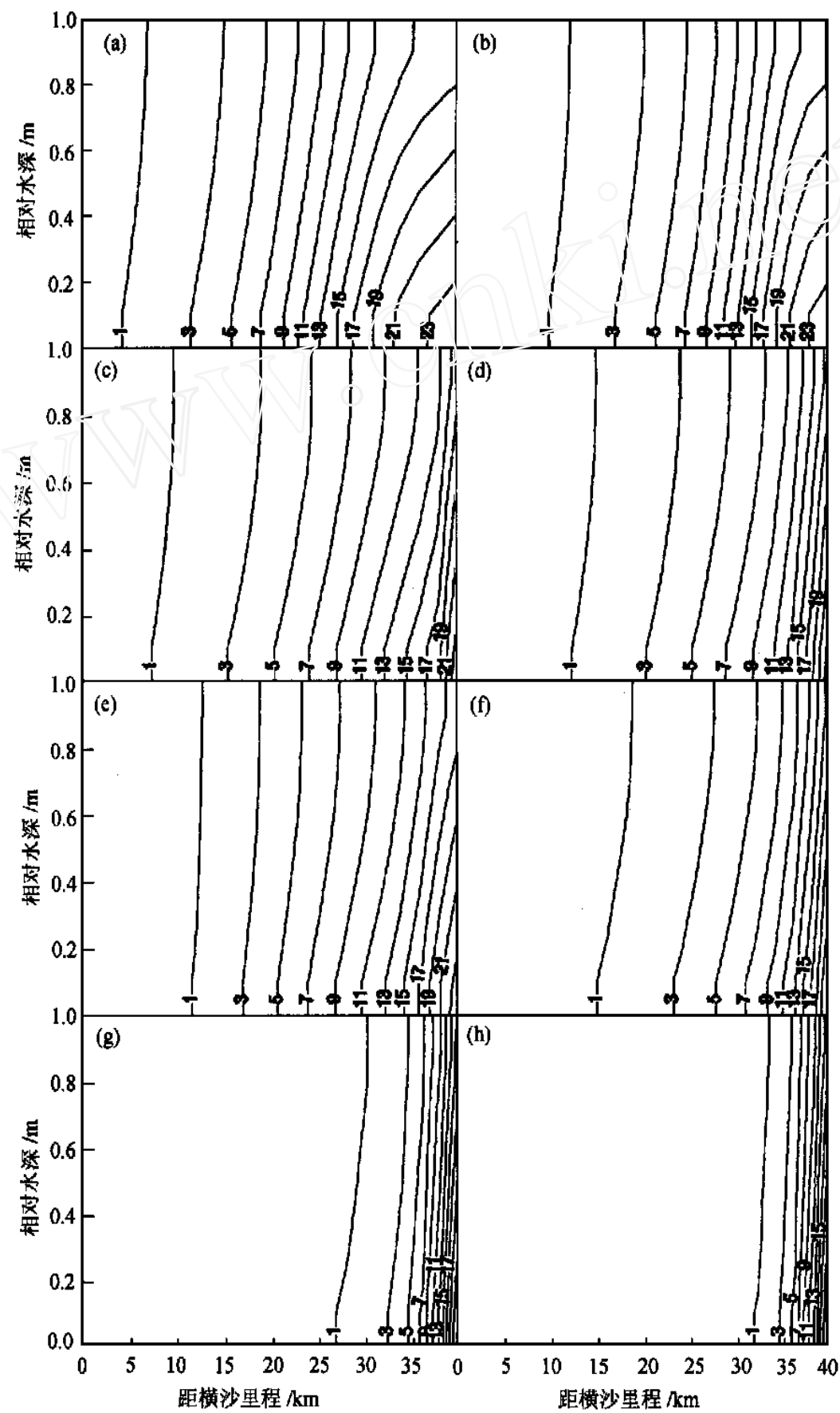

图 6 等盐度线随时间、径流、潮流的变化

(a) 涨息附近, (b) 涨急附近, (c) 落急附近, (d) (h) 落息附近

时, 因潮流增大, 盐度锋面趋向上游, 相差可达 $5 \mathrm{~km}$. 而在潮差相同时, 径流的增加, 限制了海 水的上溯, 使盐水锋面下移, 相差达 $15 \mathrm{~km}$. 因此, 径流和潮流的作用分别使盐度锋面向下移 和上溯两个相反方向发展,径流的作用比潮差更明显. 


\section{4 结论}

本文以河口水流运动的物理特征为基础, 根据振荡边界层理论进行径流潮流分解, 得到了 往复运动水流流速垂向结构, 建立了较合理的河口准二维水流盐度数学模型, 并得到长江口北 槽实测资料的验证.

应用该模型研究河口盐淡水混合过程,得到盐度分布敖径湖流变化的规律 :盐度水平分布

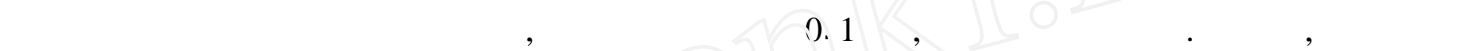
分布还因时间而异, 涨憩时梯度最小,落息时梯度最二大. 盗度垂向分布梯度沿程从小到大、再 从大到小变化, 在下游可出珿盐水棈! 的特征. 盐度锋面随径流增大而下移, 随潮流增强而上 溯. 平均盐度锋强容与河「.]掺混系数大约成线性关系.

该模型可作为进一步矿究河口泥沙输运的基础, 与以往的简化数学模型相比, 物理机理清 晰, 计算量小. 但模型也有一些局限性, 如对地形突变的局部河段 (水流运动偏离振荡边界层) 和弱混合型河口 (盐度垂向梯度大, 水流为非一维运动) 等, 计算结果会产生一定误差.

\section{参考文献}

1 茅志昌. 长江口盐水入侵锋研究. 海洋与湖沼, 1995, 26(6) : 643 649

2 Grubert J P. Interfacial mixing in stratified channel flows. J Hydr Div, ASCE, 1989 , 115(7) : $887 \sim 905$

3 Grubert J P. Interfacial mixing in estuaries and fjords. J Hydr Engrg, 1990 , 116(2) : 176 195

4 Kurup G R, Hamilton D P, Patterson J C. Modelling the effect of seasonal flow variations on the position of salt wedge in a microtidal estuary. Estuarine, Coastal and Shelf Science, 1998, 47: 191 208

5 韩曾萃, 邵雅琴, 卢祥兴. 河口咸水入侵的数学模型及物理模型预测. 第六届全国计算流体力学会议论文集, 计算流体 力学的理论、方法和应用. 北京: 科学出版社, 1992. 331 334

6 Bloss S. Lehfeldt R. Patterson J C. Modeling turbulent transport in stratified estuary. J Hydr Engrg, ASCE, 1988, 114(9) : 1 115 1133

7 Jin X, Krennburg C. Quasi-3D numerical modeling of shallowwater circulation. J Hydr Engrg, 1993 , 119(4) : 458 472

8 Irene Katopodi. Jan S Ribberink. Quasi-3D modeling of suspended sediment transport by currents and waves. Coastal Engineering, $1992,18: 83 \sim 110$

9 Collins M B , Ke X, Gao S. Tidally-induced flow structure over intertidal flats. Estuarine, Coastal and Shelf Science, 1998 , 46 : $233 \sim 250$

10 Nielsen P. Coastal Bottom Boundary Layers and Sediment Transport. Advanced Series on Ocean Engineering, Vol. 4. World Scientific , 1992. $40 \sim 52$

11 王允菊,张志忠, 黄文盛,等. 长江口南槽水化学特性与悬沙粘土矿物. 海洋通报, 1995,14(3)：106 113 